\title{
Optical Transitions and Enhanced Angular Overlap Model for the Low Symmetry Europium(III) System
}

\author{
P. Solarz* and Z. Gajek \\ Institute of Low Temperature and Structure Research, Polish Academy of Sciences, Okólna 2, \\ 50-422 Wroctaw, Poland
}

Received: September 29, 2009; Revised Manuscript Received: April 20, 2010

\begin{abstract}
Absorption, emission, and excitation spectra covering a wide energy range of $3000-58000 \mathrm{~cm}^{-1}$ for the $\mathrm{Eu}^{3+}$ ion in the low symmetry system $\mathrm{K}_{5} \mathrm{Li}_{2} \mathrm{EuF}_{10}$ have been reported. The emission spectra have been analyzed by taking into account the influence of the temperature onto the luminescence from the ${ }^{5} \mathrm{D}_{0,1,2}$ multiplets. The observed energy intervals in transition bands characteristic for the europium ion have been assigned and modeled using a multistep approach developed for this system. A risk of producing numerical artifacts in the multidimensional parameter space has been minimized by its strong reduction based on the enhanced angular overlap model and standardization of the low symmetry crystal field. The reported results are satisfactory (the final rms error of the fitting below $9.2 \mathrm{~cm}^{-1}$ ), consistent in each step of the procedure and in line with the microscopic physicochemical picture of the crystal field effective interaction.
\end{abstract}

\section{Introduction}

A single crystal of $\mathrm{K}_{5} \mathrm{Li}_{2} \mathrm{EuF}_{10}$ (KLEF) has been grown and examined in the systematic investigations of series of compounds $\mathrm{K}_{5} \mathrm{Li}_{2} \mathrm{LnF}_{10}\left(\mathrm{Ln}=\mathrm{Pr}^{3+}, \mathrm{Nd}^{3+}, \mathrm{Sm}^{3+}, \mathrm{Eu}^{3+}\right)$ to explain the phenomena of weak concentration self-quenching of luminescence of $\mathrm{Ln}^{3+}$ ions in these crystals. The compounds have been found to show exceedingly low luminescence quenching depending on the concentration of active ions. ${ }^{1-6}$ Efficient luminescence is observed even for fully concentrated material. Such a crystal is called "stoichiometric luminescence material" or self-activated compound. ${ }^{7,8}$ Characteristic regularities of the experimental data gathered during decades can be helpful in assessment of the radiative and nonradiative transitions rates within and beyond the Judd-Ofelt theory. ${ }^{9,10}$ In particular, the multiphonon relaxation rates may be predicted from so-called "energy gap law". The rate of nonradiative decay due to activator-activator interaction is less straightforward. Migration of the excitation energy and the donor-acceptor energy transfer require analysis of luminescence decay curves and their deviations from pure exponential time dependence, which are attributed to ion-ion interaction. It is accepted that the smallest possible distance between interacting ions is a factor that governs the rate of nonradiative energy transfer. The weak self-quenching of luminescence has been found in $\mathrm{K}_{5} \mathrm{Li}_{2} \mathrm{EuF}_{10},{ }^{4}$ where the emission decay curves follow a pure exponential time dependence.

The discussion of this interesting material is extended now toward possibly complete spectroscopic characterization comprising absorption, excitation spectra in the whole energy range up to the $\mathrm{f}-\mathrm{d}$ absorption edge, and luminescence including the luminescence decay characteristics. All the experimental data, new and old, are modeled then on the ground of the firm phenomenological theory. To avoid ambiguities due to the high number of parameters, certain restrictive models are applied to start with. They are based on the enhanced angular overlap model (EAOM). ${ }^{11}$

The point group symmetry of the lanthanide ion in the $\mathrm{K}_{5} \mathrm{Li}_{2} \mathrm{LnF}_{10}$ matrix is low, $C_{s}{ }^{2,3,5,12,13}$ The ground configuration

\footnotetext{
* Corresponding author. E-mail: solarz@int.pan.wroc.pl.
}

of the $\mathrm{Eu}^{3+}$ ion, $4 \mathrm{f}^{6}$, splits in this symmetry into 3003 singlets, only 65 of which form well separated bands belonging to the ${ }^{7} \mathrm{~F}_{J}(J=0-6)$ and ${ }^{5} \mathrm{D}_{J}(J=0-3)$ terms below $25000 \mathrm{~cm}^{-1}$. About 1380 further electronic levels lying below the strong $\mathrm{f}-\mathrm{d}$ absorption edge at about $58000 \mathrm{~cm}^{-1}$ form several dense, mixed- $J$ bands separated by gaps, characteristic for this specific multielectron system. On the other hand, only 160 electronic levels have been identified in this range.

Besides 20 so-called free-ion parameters (FIP), the standard phenomenological description ${ }^{14-18}$ requires 14 crystal field parameters (CFP) that represent the influence of the lattice. Apart from this discouragingly great total number of parameters (34!) and dense, mixed, multiterms bands of levels above 25000 $\mathrm{cm}^{-1}$, being indistinguishable from the symmetry point of view, one deals with unpredictable at present probabilities of the individual $\mathrm{CF}$ transitions identified in the recorded spectra beyond the most characteristic features of the multiplet-tomultiplet transitions, the specific "finger print" of the each lanthanide ion. ${ }^{16-19}$

All these difficulties make the conventional phenomenological analysis for the europium ion in low symmetry systems rather risky and, in consequence, rarely attempted. Those trails that can be found in the literature have been based on certain additional constrains or information including

- simultaneous analysis of several lanthanide elements doped in a given host matrix ${ }^{16}$

- initial CF parameters taken from another element of the series $^{20,21}$

- compounds with multiple sites for the metal ion ${ }^{21,22}$

- restriction of investigations to compounds of higher than $C_{1}, C_{2}, C_{s}$, or $C_{i}$ actual or approximate symmetries ${ }^{16,20,22,23}$

- spectra depended on the polarization of light ${ }^{20}$

- simultaneous investigation of intensities ${ }^{20,22}$

- initial CF parameters from ab initio calculations ${ }^{16,22}$

- simplified phenomenological models ${ }^{24}$

Our multistep procedure is constrained by the regularity of FIP ${ }^{15-17,25}$ ab initio calculations of certain contributions to CFP, and the mentioned simplified CF model called the enhanced angular overlap model EAOM. ${ }^{11}$ 
The main idea of EAOM is simple and quite common in physics and chemistry: to excerpt everything that can be calculated directly and parametrize the remainder. Following this idea, the total CF effective potential is divided into two parts: the adjustable one based on the ordinary angular overlap model (AOM $)^{26-28}$ and the off-AOM part (OA) comprising these contributions to the effective crystal field potential that do not satisfy the angular overlap model assumptions. ${ }^{29}$ For f-electron systems like $\mathrm{K}_{5} \mathrm{Li}_{2} \mathrm{EuF}_{10}$ the $\mathrm{OA}$ part is rather small, especially for the four and six rank parameters, but not so small as to be ignored. ${ }^{11,29-31}$

Expecting higher accuracy of the readout of the experimental level positions than EAOM assures, it is desirable to apply the general, one-electron $\mathrm{CF}$ parametrization with the assignation of levels and the initial parameters determined within the EAOM phase. As we will see, improvement of the results justifies such a refinement.

Contrary to the common descent of symmetries technique (DST), ${ }^{16,20,22,23}$ EAOM requires certain auxiliary calculations. Nevertheless, its application does not depend on existence of approximate symmetries and allows one to avoid uncertainty due to possible supersensitivity of the CF parameters to even slight distortion of the coordination polyhedron. ${ }^{23}$ Since an approximate symmetry does exist for the compound under consideration $\left(D_{2 d}\right)$ it is possible to face the two methods.

The conventional CF parametrization in the case of the $C_{s}$ symmetry is not unique. Its standardization described in the Results and Discussion allows one to avoid confusing ambiguities and to equate different $\mathrm{CF}$ parameter sets genuinely. Thus, similarly as in ref 24 , both the (E)AOM results and the refined ones are kept in the same standardized form.

The paper is organized as follows. The experimental details set forth in next section are followed by brief description of the phenomenological model. The spectra are presented, analyzed, modeled, and discussed in the Results and Discussion. Concluding remarks close the paper.

\section{Experimental Section}

Single crystal of $\mathrm{K}_{5} \mathrm{Li}_{2} \mathrm{EuF}_{10}$ (KLEF) was grown by the vertical Bridgman method in graphite crucibles made from high quality IG-110 purified graphite. The crystal was $50 \mathrm{~mm}$ long and $5 \mathrm{~mm}$ in diameter, colorless, and transparent. For low temperature measurements a continuous flow helium cryostat (Oxford model CF 1204) equipped with a temperature controller was used.

Luminescence and excitation spectra were gathered with a Hamamatsu R-955 PMT using one channel 750 focal length a Dongwoo Optron spectrophotometer (model DM711). To excite the sample, a $180 \mathrm{~W}$ ozone-free, Dongwoo Optron lamp were used. The High-UV-VUV excitation spectrum was recorded using a setup of SUPERLUMI station at HASYLAB, DESY in Hamburg, Germany. ${ }^{32}$ In this case the response of the system was corrected with sodium salicylate, which emits a number of visible photons in linear proportion to a number of UV-VUV photons used for excitation. For absorption measurements the Cary 5E UV-vis-NIR spectrophotometer was used.

In luminescence decay time measurements, short (4 ns) pulses delivered by an optical parametric oscillator OPO (Continuum, Surelite I) pumped by the third harmonic of Nd:YAG laser were used to excite directly luminescence levels. The decay signal was detected, averaged, and stored with a Tektronix TDS 3052 digital oscilloscope; all decay data were composed of 10000 points. The fits of experimental decay curves were done using the Microcal Origin v5.0 software; the amplitude of the curves was calibrated to 10 .

\section{Phenomenological Model}

An effective Hamiltonian describing $N$-electron localized states in a transparent, optically active solid ${ }^{14-18}$ is obtained by a projection of the true Hamiltonian on the subspace spanned by the ground state configuration $\mathrm{f}^{N}$ wave functions. It is a common (and good) approximation to assume the same radial part for all the one-electron orbitals, the truncated function basis is constructed with. This assumption allows integration of the Schrödinger equation over the radial coordinate to get Hamiltonian dependent merely on the spin and angular coordinates of electrons. It is natural to distinguish in the resulting effective Hamiltonian $\hat{H}$ the "free-ion" $\hat{H}^{\mathrm{FI}}$ and the crystal field $\hat{H}^{\mathrm{CF}}$ parts:

$$
\hat{H}=\hat{H}^{\mathrm{FI}}+\hat{H}^{\mathrm{CF}}
$$

$\hat{H}^{\mathrm{FI}}$ represents several intra-atomic, spherically symmetric interactions, the most important of which is the interelectron Coulomb repulsion and the spin-orbit (SO) interaction controlled with the Slater integrals $F^{k}$ and SO coupling constant $\zeta_{4 \mathrm{f}}$, respectively. In the commonly accepted form, the free-ion Hamiltonian,

$$
\begin{aligned}
& \hat{H}^{\mathrm{FI}}=\sum_{k=0,2,4,6} F^{k} \hat{f}_{k}+\xi_{4 f} \hat{A}_{S O}+ \\
& \alpha \hat{L}(\hat{L}+1)+\beta \hat{G}\left(G_{2}\right) \gamma \hat{R}\left(R_{7}\right)+ \\
& \sum_{k=2,3,4,6,7,8} T^{k} \hat{t}_{k}+\sum_{k=0,2,4} M^{k} \hat{m}_{k}+\sum_{k=2,4,6} P^{k} \hat{p}_{k}
\end{aligned}
$$

includes also certain higher order terms: ${ }^{14-18,33}$ Namely, the twoand three-electron configuration interaction corrections with Trees parameters $\alpha, \beta$, and $\gamma$ and Judd integrals $T^{k}(k=2,3$, $4,6,7,8)$, respectively, and the further relativistic corrections of higher than SO interaction order with the $P^{k}(k=2,4,6)$ and $M^{k}(k=0,2,4)$ parameters. The corresponding operators $\hat{f}_{k}, \hat{A}_{\mathrm{SO}}, \hat{G}\left(G_{2}\right), \hat{L}, \hat{R}\left(R_{7}\right), \hat{t}_{k}, \hat{m}_{k}$, and $\hat{p}_{k}$ acting on angular (and spin) coordinates of electrons have their usual meaning; see refs 14-18 and 33 for further details.

The effective crystal-field interaction $\hat{H}^{\mathrm{CF}}$ must be invariant under transformations of the point group. It is usually expanded in terms of the spherical tensor operators $\hat{C}_{q}^{(k)}(i) .{ }^{14,16}$ For the $C_{s}$ $\equiv C_{1 h}$ symmetry and the coordinate system coinciding with the unit cell axes in which the $y$ axis is perpendicular to the reflection plane, it takes the form

$$
\hat{H}^{\mathrm{CF}}=\sum_{i, k} \sum_{q=0}^{k} B_{k q} \hat{C}_{q}^{(k)}(i)
$$

where $i$ runs over the electrons and $k=2,4,6$. Equation 3 represents one of three equivalent parametrizations in which all the 15 crystal field parameters $B_{k q}$ are real. In the two alternative parametrizations (not considered here) some of the parameters are imaginary or complex depending on orientation of the coordinate system with respect to the symmetry plane. ${ }^{34,35}$ These three settings may be related to three solutions of the set of equations

$$
\begin{aligned}
B_{21} & =0 \\
B_{2-1} & =0
\end{aligned}
$$


with respect to the Euler angles rotating the coordinate system. ${ }^{36}$ The possibility of elimination of one of the parameters appears as a consequence of freedom in orientation of the coordinate system with respect to the reflection plane. Thus, if the expansion (3) with all the real parameters is to be used in the fitting, the number of these varied simultaneously has to be reduced to 14 , preferably by excluding $B_{21} \cdot{ }^{36}$

The parameters in particular settings can be transformed into each other by respective rotations of the coordinate system. Unfortunately, there are further ambiguities: four equivalent parameter sets for each of the setting. In the present case of the expansion (3) they can be generated by the rotations of the coordinate system by 90 and $180^{\circ}$ about the $y$ and $z$ axes, respectively.

Two different sets of parameters to be compared must be expressed in the same, one of the four equivalent, coordinate systems. It can be fixed according to the standardization scheme based on the following two rules:

(i) The maximum of the absolute value of the $B_{20}$ parameter, the idea suggested first by Rudowicz ${ }^{37}$ and developed by Mulak. ${ }^{38}$ This removes the ambiguity due to the rotation by $90^{\circ}$ about the $y$ axis.

(ii) The maximum of the correlation factor $c$ defined as the normalized "scalar product" of the two sets of parameters, e.g., $A$ and $B$, of $q$ odd

$$
c=\sum_{k=4,6} \frac{1}{2 k+1} \sum_{q \text { odd }}^{k-1}\left(B_{k q}\right)^{A}\left(B_{k q}\right)^{B}
$$

The correlation factor $c$ resembles the reliability factor introduced by Faucher and Garcia to rate the convergence of the experimental (phenomenological) and theoretical (ab initio) sets of CF parameters. ${ }^{30}$ If the sets A and B are the same, $c$ represents the $q$-odd component of the square of $\mathrm{CF}$ strength parameter (17).$^{39,40}$ This condition removes the ambiguity due to the rotation by $180^{\circ}$ about the $z$ axis that inverts the signs of the $q$-odd parameters.

The algebraic formulas for the rotation matrices of rank 2, 4, and 6 according to which the $B_{k q}$ parameters are transformed can be found in ref 41 .

The CF Hamiltonian in the EAOM approach is divided into parts:

$$
\hat{H}^{\mathrm{CF}}=\hat{V}^{\mathrm{AOM}}+\hat{V}^{\mathrm{OA}}
$$

The OA part can be written in the same form as the total $\mathrm{CF}$ potential (3).

$$
\hat{V}^{\mathrm{OA}}=\sum_{i, k} \sum_{q=0}^{k}\left(B_{k q}\right)_{\mathrm{OA}} \hat{C}_{q}^{(k)}(i)
$$

it includes point charge contribution of further neighbors of the metal ion as well as point dipoles and quadruples induced on all ions in the lattice. We neglect the renormalization of the OA terms, ${ }^{11,28,29,31}$ except for the shielding that may not be ignored in the case of f-electron systems. ${ }^{42}$ Despite strong shielding, the electrostatic contributions to the OA terms remain essential. ${ }^{11,29,31}$ The parameters $\left(B_{q}^{k}\right)_{\mathrm{OA}}$ have been calculated using formulas given in refs 11 and 31, the crystallographic data from refs $2,3,5$, and 12 , the dipole and quadrupole polarizabilities of the ions from ref 43 , and the Sternheimer shielding factors
TABLE 1: Coordination Factors $W_{k q}^{\mu}$ and the Off-AOM (OA) CFP

\begin{tabular}{rrrrrr}
\hline & & \multicolumn{4}{c}{$W_{k q}^{u}$} \\
\cline { 3 - 5 }$k$ & $q$ & $\mu=0$ & $\mu=1$ & $\mu=2$ & $\left(B_{k q}\right)_{\mathrm{OA}}$ \\
\hline 2 & 0 & 0.461 & 0.743 & 0.000 & -236 \\
2 & 1 & -0.298 & -0.476 & 0.000 & 158 \\
2 & 2 & 0.066 & 0.100 & 0.000 & 2 \\
4 & 0 & -0.110 & -0.043 & 0.375 & 50 \\
4 & 1 & 0.276 & 0.095 & -0.699 & 14 \\
4 & 2 & 0.578 & 0.190 & -1.288 & 95 \\
4 & 3 & 1.360 & 0.450 & -3.121 & 154 \\
4 & 4 & 1.873 & 0.615 & -4.200 & 270 \\
6 & 0 & 0.381 & -0.581 & 0.239 & 14 \\
6 & 1 & 1.697 & -2.591 & 1.068 & 36 \\
6 & 2 & -0.699 & 1.068 & -0.441 & -22 \\
6 & 3 & 1.297 & -2.014 & 0.852 & 3 \\
6 & 4 & -0.076 & 0.113 & -0.044 & -1 \\
6 & 5 & -0.890 & 1.358 & -0.560 & -19 \\
6 & 6 & 0.587 & -0.882 & 0.354 & 19
\end{tabular}

from ref 42. The computer program CHLOE by Faucher ${ }^{44}$ for calculations of the lattice electrostatic contributions, adopted by one of the authors (Z.G.), has been employed. The results are shown in Table 1.

The angular overlap model (AOM) is a simplified phenomenological approach based on certain restrictive assumptions:

(i) $\mathrm{CF}$ potential $V^{\mathrm{AOM}}$ is superposition of independent contributions, effective potentials $v_{t}$ generated by the nearest neighbors (ligands);

(ii) local symmetry of the potential $v_{t}$ is axial along the metal-ligand axis. Each AOM parameter $e_{\mu}^{t}$ is defined as a respective matrix element of single-ligand potential $v_{t}$ in local coordinate system $t$ in which it is, according to (ii), diagonal in the coordinate system where the $z$ axis is directed along the metal-ligand $t$ axis:

$$
e_{\mu}^{t}=\left\langle\mu\left|v_{t}\right| \mu\right\rangle_{t}
$$

where $\mu=0(\sigma), 1(\pi), 2(\delta)$ denotes the magnetic quantum number of the $4 \mathrm{f}$-electron in this local coordinate system. The matrix elements of AOM part of $\mathrm{CF}$ in global coordinate system are parametrized as follows:

$$
\left\langle m|V| m^{\prime}\right\rangle=\sum_{t, \mu} D_{\mu m}^{(3)^{*}}\left(0, \Theta_{t}, \Phi_{t}\right) D_{\mu m^{\prime}}^{(3)}\left(0, \Theta_{t}, \Phi_{t}\right) e_{\mu}^{t}
$$

where $D_{\mu m}^{(3)}\left(0, \Theta_{t}, \Phi_{t}\right)$ is the matrix element of the irreducible representation $D^{(3)}$ of the rotation group and $R_{t}, \Theta_{t}$, and $\Phi_{t}$ are the angular (global) coordinates of the ligand $t$.

Equation 9 relates the matrix elements of the $\mathrm{CF}$ potential to the intrinsic parameters describing the individual metal-ligand pairs. The conventional CFP can be calculated from set of linear equations:

$$
B_{k q}=\sum_{\mu} W_{k q}^{\mu} e_{\mu}
$$

where 


$$
\begin{aligned}
W_{k q}^{\mu}= & \frac{2 k+1}{7}\left[\left(\begin{array}{lll}
3 & k & 3 \\
0 & 0 & 0
\end{array}\right)\right]^{-1}(-1)^{\mu}\left(2-\delta_{\mu 0}\right) \times \\
& \left(\begin{array}{ccc}
3 & k & 3 \\
-\mu & 0 & \mu
\end{array}\right) \sum_{t} C_{q}^{(k)^{*}}\left(\Theta_{t}, \Phi_{t}\right) s_{\mu}^{t}
\end{aligned}
$$

$$
s_{\mu}^{t}=\frac{e_{\mu}^{t}}{e_{\mu}}
$$

and $(:::)$ are the $3 j$ symbols. The $e_{\mu}$ parameters in eqs 11 and 12 are the mean values of the AOM parameters averaged over $t$. Their introduction is necessary if nonequivalent ligands occur. The $W_{k q}^{u}$ coefficients absorb all information about the geometry of the coordination polyhedron whereas the ratios $s_{u}^{t}$ encode differences in the $\mathrm{AOM}$ parameters due to the various $\mathrm{ML}_{t}$ distances. In principle, the distance dependence of $s_{\mu}^{t}$ has the exponential character, ${ }^{29}$ yet, within a limited range, it can be approximated by the power function:

$$
s_{\mu}^{t}=\left(\frac{R}{R_{t}}\right)^{\alpha_{\mu}}
$$

with the power exponents $\alpha_{\mu}$ taking values from 4.3 to 8.9. . $^{28,29,31}$

Coordination factors shown in Table 1 together with the OA CFP have been calculated using the crystallographic data from refs 2, 4, and 5 (see next Results and Discussion). The model can be further simplified, first by ignoring the smallest AOM parameter $e_{\delta}$ and second by keeping the ratio of the two remaining parameters fixed: ${ }^{29,31}$

$$
r=\frac{e_{\pi}}{e_{\sigma}} \approx 0.39
$$

Thus, we obtain the one-parameter CF model denoted hereafter as EAOM-I to distinguish it from the standard three-parameter EAOM. For the approximate symmetry $D_{2 d}$ (see section "Crystal Structure" for details) the expansion (3) reduces to five terms with the only nonzero CF parameters $B_{20}, B_{40}, B_{44}, B_{60}$, and $B_{64}$.

\section{Results and Discussion}

Crystal Structure. According to refs 2, 4, and 5, the $\mathrm{K}_{5} \mathrm{Li}_{2} \mathrm{EuF}_{10}$ crystals are orthorhombic (space group $D_{2 h}^{16}$, Pnma). For lanthanide $\mathrm{La}-\mathrm{Gd}$ the cell parameters have been found to be $a=20.599 \AA, b=7.752 \AA$, and $c=6.879 \AA$. $^{6}$ The crystal structure is built from layers perpendicular to the $a$ axis, formed by $\mathrm{EuF}_{8}$ dodecahedra and $\mathrm{LiF}_{4}$ tetrahedra. $\mathrm{Eu}^{3+}$ and $\mathrm{Li}^{+}$ions occupy sites with $C_{s}$ point symmetry whereas potassium ions and fluorine occupy sites with $C_{s}$ and $C_{1}$ symmetry. The $\mathrm{EuF}_{8}$ polyhedra do not share fluorine ions. The closest lanthanide ions are separated by about $6.5 \AA$; thus the exchange interactions between Eu ions may be neglected.

The coordination of the $\mathrm{Eu}^{3+}$ ion is presented in Figure 1. The detailed information about the $\mathrm{K}_{5} \mathrm{Li}_{2} \mathrm{EuF}_{10}$ crystal structure has been given recently by Gagor. ${ }^{13}$

The approximate coordination polyhedron of europium, point group symmetry $D_{2 d}$, consists of two interpenetrating fluorine tetrahedra, elongated and flattened one with two different metal-ligand distances, 2.450 and $2.382 \AA$, respectively. The deviation of the idealized fluorine positions varies from 0.038 to $0.110 \AA$, and its mean square value amounts to $0.075 \AA$.

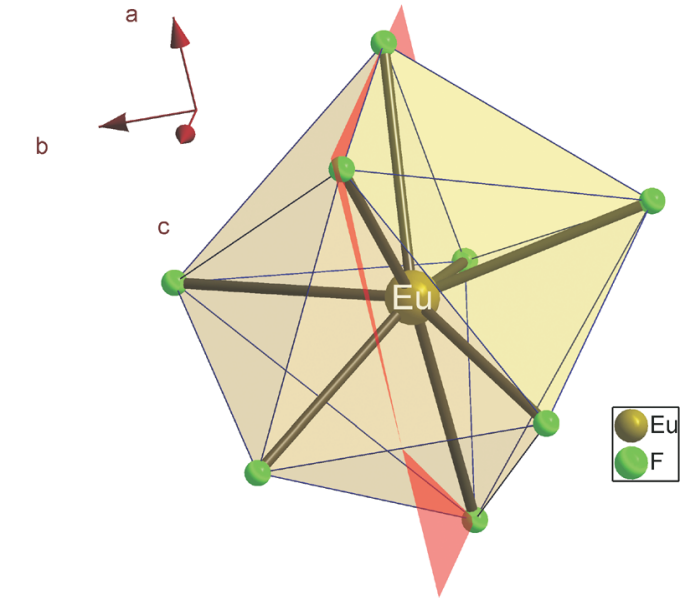

Figure 1. First coordination sphere of $\mathrm{Eu}^{3+}$ ion in the $\mathrm{K}_{5} \mathrm{Li}_{2} \mathrm{EuF}_{10}$ crystal.

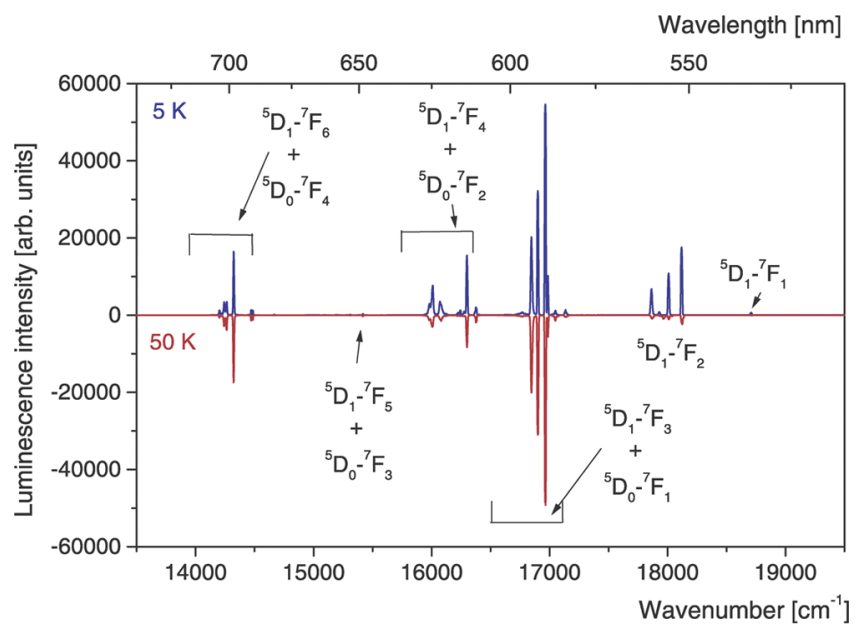

Figure 2. Emission spectra of $\mathrm{K}_{5} \mathrm{Li}_{2} \mathrm{EuF}_{10}$ recorded at 5 (blue) and 50 (brown) $\mathrm{K}$ upon excitation at $393 \mathrm{~nm}$. For better visualization of this picture, the $\mathrm{K}_{5} \mathrm{Li}_{2} \mathrm{EuF}_{10}$ spectrum recorded at $50 \mathrm{~K}$ is shown inverted.

The main 2-fold symmetry axis lies in the $x z$ plane at the angle $-24.9^{\circ}$ to the crystallographic $z$ axis.

Spectroscopic Properties. Intensity of Spectroscopic Transitions. The emission from $\mathrm{Eu}^{3+}$ ions even in stoichiometric crystal $\mathrm{K}_{5} \mathrm{Li}_{2} \mathrm{EuF}_{10}$ originates mainly from the ${ }^{5} \mathrm{D}_{0}$ and ${ }^{5} \mathrm{D}_{1}$ multiplet with addition of emission from the ${ }^{5} \mathrm{D}_{2}$ multiplet. For dilution with La crystals, the emission from the ${ }^{5} \mathrm{D}_{3}$ multiplet has been observed also (see ref 4). Moreover, the lifetimes of the ${ }^{5} \mathrm{D}_{0}$ and ${ }^{5} \mathrm{D}_{1}$ excited states, recorded at $5 \mathrm{~K}$, are of the same order of magnitude that complicates the assignment of emission lines situated in the same spectral area corresponding to the transitions ${ }^{5} \mathrm{D}_{0} \rightarrow{ }^{7} \mathrm{~F}_{J}$ and ${ }^{5} \mathrm{D}_{1} \rightarrow{ }^{7} \mathrm{~F}_{J+2}$. They have been assigned by careful analysis of possible energy level schemes including those discussed in the earlier studies ${ }^{4}$ and the finding that the lifetime of the excited ${ }^{5} \mathrm{D}_{0}$ multiplet does not depend on temperature as opposed to the lifetime of the ${ }^{5} \mathrm{D}_{1}$ one.

Figure 2 shows emission spectra of the $\mathrm{K}_{5} \mathrm{Li}_{2} \mathrm{EuF}_{10}$ crystal at $5 \mathrm{~K}$ (normal direction) and $50 \mathrm{~K}$ (inverted for better readability) upon excitation at $394 \mathrm{~nm}$. All other parameters were kept the same. The difference between the luminescence spectra recorded at 5 and $50 \mathrm{~K}$ most clearly seen in the region near $18700 \mathrm{~cm}^{-1}$ depends on temperature quenching of luminescence originated from the ${ }^{5} \mathrm{D}_{1}$ multiplet. These spectra are mainly composed of transitions at about $18000,17000,16000$, and $14500 \mathrm{~cm}^{-1}$, which are allowed by the selection rules. Other transitions at 


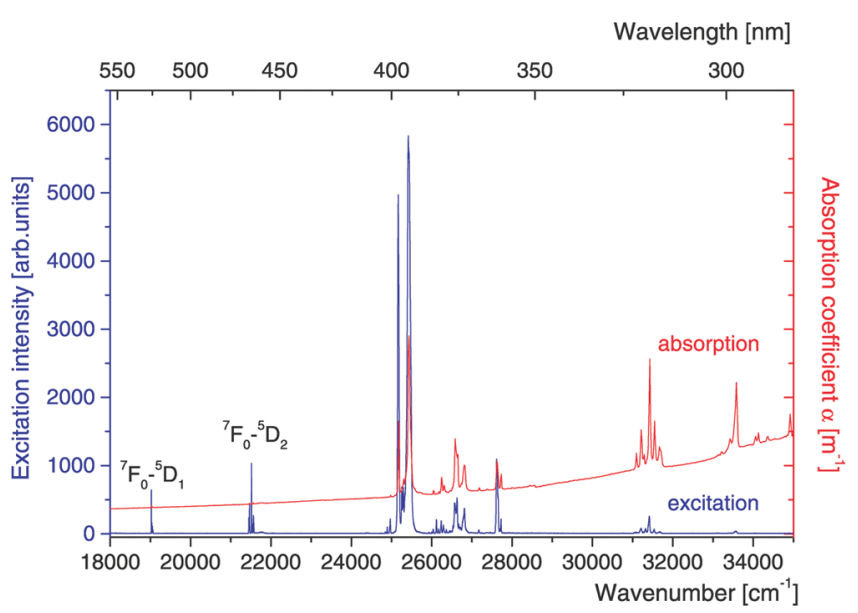

Figure 3. Vis-UV excitation spectrum of luminescence observed at $592 \mathrm{~nm}$ (blue) and absorption spectrum of KLEF (red) both recorded at $5 \mathrm{~K}$.

about 15500 and $18700 \mathrm{~cm}^{-1}$ have much lower intensity. The ${ }^{5} \mathrm{D}_{0} \rightarrow{ }^{7} \mathrm{~F}_{0}$ transition, being forbidden in the limit of pure SLJ states by both magnetic dipole and electric dipole selection rules, is situated at $17283 \mathrm{~cm}^{-1}$. The ${ }^{5} \mathrm{D}_{0} \rightarrow{ }^{7} \mathrm{~F}_{2}$ transition was found to be very weak. Its intensity is inferior even to those from ${ }^{5} \mathrm{D}_{1}$ $\left(\sim 19000 \mathrm{~cm}^{-1}\right)$, which are of magnetic origin. As for the hypersensitive transitions $(\Delta J=2)$, this observation is quite unusual, taking into account the low europium site symmetry. ${ }^{45-48}$

We compared the intensity of luminescence of our stoichiometric $\mathrm{K}_{5} \mathrm{Li}_{2} \mathrm{EuF}_{10}$ with another fluoride system, $\mathrm{KY}_{3} \mathrm{~F}_{10}: \mathrm{Eu}^{3+}$, in which $\mathrm{Eu}^{3+}$ ions occupy a site of $C_{4 v}$ symmetry doped only with 1 at. $\%$ of europium. ${ }^{49}$ The main divergence was noticed in the intensities of the hypersensitive transition ${ }^{5} \mathrm{D}_{0} \rightarrow{ }^{7} \mathrm{~F}_{2}$ with relation to the magnetic dipole transition ${ }^{5} \mathrm{D}_{0} \rightarrow{ }^{7} \mathrm{~F}_{1}$. Contrary to common observation, ${ }^{50}$ the ratio was greater in the case of the compound of higher symmetry. The observation was confirmed by the lifetimes of the ${ }^{5} \mathrm{D}_{0}$ multiplet. The two main pathways of depopulation of this multiplet go through transitions to the ${ }^{7} \mathrm{~F}_{2}$ and ${ }^{7} \mathrm{~F}_{1}$ multiplets. The transition to ${ }^{7} \mathrm{~F}_{1}$ depends only on the refractive index of the matrix. For $\mathrm{KY}_{3} \mathrm{~F}_{10}$ the refractive index $n$ is $1.490^{49}$ whereas for $\mathrm{K}_{5} \mathrm{Li}_{2} \mathrm{EuF}_{10}$ it is equal to 1.40 . In spite of this, in lower symmetry $\mathrm{K}_{5} \mathrm{Li}_{2} \mathrm{EuF}_{10}$ we observed the lifetime of the ${ }^{5} \mathrm{D}_{0}$ multiplet twice as long as in $\mathrm{KY}_{3} \mathrm{~F}_{10}$ : $\mathrm{Eu}^{3+} .^{51}$ With addition of sensitizers like $\mathrm{Tb}^{3+}, \mathrm{Pr}^{3+}$, or $\mathrm{Gd}^{3+}$ ions this compound might be used as a new phosphor for mercury free lamps. ${ }^{52}$

The absorption spectrum presented in Figure 3 was recorded with a good-quality single crystal. The measurements were performed with a stoichiometric KLEF sample to exclude any ambiguity due to the $\mathrm{Eu}^{3+}$ location, likely to occur when doped crystals are considered. Note that the hypersensitive transition ${ }^{7} \mathrm{~F}_{0} \rightarrow{ }^{5} \mathrm{D}_{2}$ is hardly visible in this pattern.

The excitation spectrum was not calibrated for the spectral response; therefore the intensity of the ${ }^{7} \mathrm{~F}_{0} \rightarrow{ }^{5} \mathrm{D}_{2}$ transitions is slightly bigger than for ${ }^{7} \mathrm{~F}_{0} \rightarrow{ }^{5} \mathrm{D}_{1}$ ones. The excitations ${ }^{7} \mathrm{~F}_{0} \rightarrow$ ${ }^{5} \mathrm{D}_{1}$ are situated at 19025,19037 , and $19053 \mathrm{~cm}^{-1}$. In this band the additional weak line $19065 \mathrm{~cm}^{-1}$ is seen. Since its intensity decreases clearly with temperature, we propose to assign it to a phonon. The unusually weak lines located at 21 449, 21 467, 21 516, 21 519, and $21565 \mathrm{~cm}^{-1}$ have been attributed to the five Stark components of the mentioned ${ }^{7} \mathrm{~F}_{0} \rightarrow$ ${ }^{5} \mathrm{D}_{2}$ transition. The additional weak line at $21542 \mathrm{~cm}^{-1}$ has been considered to be a phonon excitation. The intensity of the subsequent ${ }^{7} \mathrm{~F}_{0} \rightarrow{ }^{5} \mathrm{D}_{3}$ transitions is very weak again, but this time in accordance with the selections rules. As for other

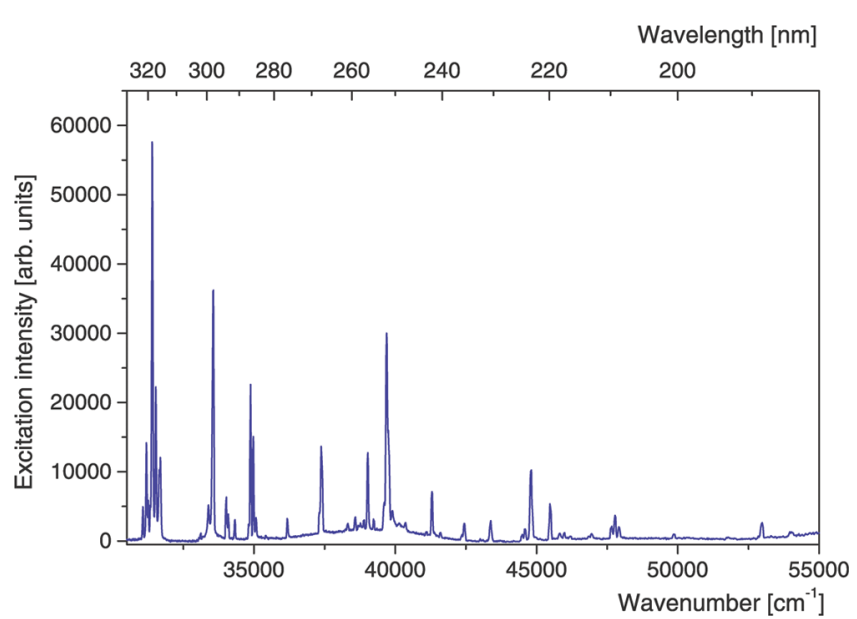

Figure 4. UV-VUV excitation spectrum of luminescence from 592 $\mathrm{nm}$ (red) recorded at $10 \mathrm{~K}$.

europium doped materials, strong lines appear at about 25000 $\mathrm{cm}^{-1}$. They represent the ${ }^{7} \mathrm{~F}_{0} \rightarrow{ }^{5} \mathrm{~L}_{6}$ transitions.

Figure 4 presents corrected for the photons flux excitation spectrum in the range $30500-55000 \mathrm{~cm}^{-1}$ recorded with synchrotron radiation. For detailed positions of observed lines, see Table 2 and Table S1 in Supporting Information.

Several lines seen in the spectra were not included into the model calculations. Their intensity is very weak and the origin is not quite clear. In VUV excitation spectra (Figure 4) one can observe a broad background band near $40000 \mathrm{~cm}^{-1}(250 \mathrm{~nm})$. Such a band has been assigned commonly in literature to $\mathrm{O}^{2-}$ $\rightarrow \mathrm{Eu}^{3+}$ charge transfer transitions. ${ }^{53-55}$ Probably additional lines originate from the hydrated surface phase of the slightly hygroscopic crystal. Therefore, some traces of another phase that contains oxygen groups could not be excluded. Additional satellite lines have been observed also in the isostructural crystal, $\mathrm{K}_{5} \mathrm{Li}_{2} \mathrm{PrF}_{10},{ }^{1,3}$ however, the authors have not resolved their origin.

On the basis of data presented in Figures 2-4 (emission, absorption and excitation spectra), the experimental positions of Stark sublevels were gathered for theoretical analysis. Certainly, due to the restrictive selection rules (and accidentally vanishing reduced matrix elements), only a small fraction of the electronic levels could be observed, especially in the case of multiplets labeled by higher values of $J$.

Dynamics of Excited State and Self-Quenching of Luminescence. As mentioned earlier, the lifetimes of the ${ }^{5} \mathrm{D}_{0}$ multiplet in KLEF do not depend on temperature. It has been presented that in diluted with $\mathrm{La}^{3+}$ crystals the ${ }^{5} \mathrm{D}_{0}$ lifetime is about 12 $\mathrm{ms}$ up to concentration of $\mathrm{Eu}^{3+}$ at the level 50 at. \%. For higher atomic concentrations it is shortened to about $9.5 \mathrm{~ms}$. Shortening of the ${ }^{5} \mathrm{D}_{0}$ lifetime in crystals containing high $\mathrm{Eu}^{3+}$ ions concentration is quite common. It has been attributed to the migration-accelerated energy transfer to unintentional traps rather than to activator-activator interactions. ${ }^{4}$

Figures 5 and 6 present the influence of temperature on the lifetime of the ${ }^{5} \mathrm{D}_{1}$ and ${ }^{5} \mathrm{D}_{2}$ multiplets, respectively. Analysis of the results shown in Figure 5 indicates that the lifetime of the ${ }^{5} \mathrm{D}_{1}$ multiplet of $\mathrm{Eu}^{3+}$ in KLEF shortens exponentially and strongly in the region $5-30 \mathrm{~K}$ from 3500 to $2500 \mu$ s. From 35 to $75 \mathrm{~K}$ the lifetimes are shortened almost linearly to $2200 \mu \mathrm{s}$. Above this temperature the shortening of lifetimes of the ${ }^{5} \mathrm{D}_{1}$ multiplet becomes again exponential. Shortening of the ${ }^{5} \mathrm{D}_{1}$ lifetime is in accordance with data presented in Figure 2. The evolution of the ${ }^{5} \mathrm{D}_{2}$ lifetime with temperature shown in Figure 
TABLE 2: List of the Spectroscopic Lines Observed in Absorption (abs), Emission $\left({ }^{5} D_{0},{ }^{5} D_{1},{ }^{5} D_{2}\right)$, and Excitation (exc) Measurements and Their Intensities (Respectively) below $21600 \mathrm{~cm}^{-1 a}$

\begin{tabular}{|c|c|c|}
\hline energy $\left(\mathrm{cm}^{-1}\right)$ & origin & intensity $^{b}$ \\
\hline 313 & ${ }^{5} D_{0},{ }^{5} D_{1}$ & vs, w \\
\hline 377 & ${ }^{5} D_{1}$ & vs \\
\hline 429 & ${ }^{5} \mathbf{D}_{0},{ }^{5} \mathbf{D}_{1}$ & $\mathbf{s}, \mathbf{v w}$ \\
\hline 900 & ${ }^{5} \mathbf{D}_{0},{ }^{5} \mathbf{D}_{1}$ & $\mathbf{m}, \mathbf{s}$ \\
\hline 1010 & ${ }^{5} \mathbf{D}_{1}$ & $\mathbf{s}$ \\
\hline 1055 & ${ }^{5} \mathbf{D}_{0},{ }^{5} \mathbf{D}_{1}$ & $\mathbf{w}, \mathbf{v w}$ \\
\hline 1089 & ${ }^{5} D_{1}$ & $\mathbf{w}$ \\
\hline 1155 & ${ }^{5} \mathbf{D}_{0},{ }^{5} \mathbf{D}_{1}$ & $\mathbf{w}, \mathbf{m}$ \\
\hline 1832 & ${ }^{5} D_{0}$ & vw \\
\hline 1858 & ${ }^{5} D_{0}$ & $\mathbf{w}$ \\
\hline 1884 & ${ }^{5} \mathbf{D}_{0},{ }^{5} \mathbf{D}_{1}$ & $\mathbf{v w}, \mathbf{w}$ \\
\hline 1905 & ${ }^{5} \mathbf{D}_{0},{ }^{5} \mathbf{D}_{2}$ & vw, vw \\
\hline 1970 & ${ }^{5} D_{0},{ }^{5} D_{1}$ & $\mathbf{w}, \mathbf{m}$ \\
\hline 2006 & ${ }^{5} \mathbf{D}_{0},{ }^{5} \mathbf{D}_{1}$ & $\mathbf{v w}, \mathbf{w}$ \\
\hline 2035 & ${ }^{5} D_{1}$ & $\mathbf{s}$ \\
\hline 2719 & ${ }^{5} D_{1}$ & $\mathbf{s}$ \\
\hline $2743^{c}$ & ${ }^{5} D_{0},{ }^{5} D_{l}$ & $v w, w$ \\
\hline 2776 & ${ }^{5} \mathbf{D}_{0},{ }^{5} \mathbf{D}_{1}$ & $\mathbf{w}, \mathbf{w}$ \\
\hline 2795 & ${ }^{5} \mathbf{D}_{0},{ }^{5} \mathbf{D}_{1},{ }^{5} \mathbf{D}_{2}$ & $\mathbf{m}, \mathbf{w}, \mathbf{v w}$ \\
\hline 2806 & ${ }^{5} \mathbf{D}_{0},{ }^{5} \mathbf{D}_{1}$ & $\mathbf{m}, \mathbf{w}$ \\
\hline 2919 & ${ }^{5} \mathrm{D}_{0}$ & vw \\
\hline 2954 & ${ }^{5} D_{0},{ }^{5} D_{1}$ & $\mathbf{s}, \mathbf{m}$ \\
\hline 3014 & ${ }^{5} \mathbf{D}_{0},{ }^{5} \mathbf{D}_{1}$ & $\mathbf{m}, \mathbf{s}$ \\
\hline 3034 & ${ }^{5} \mathbf{D}_{0},{ }^{5} \mathbf{D}_{1}$ & $\mathbf{m}, \mathbf{m}$ \\
\hline 3815 & ${ }^{5} \mathrm{D}_{1}$ & vw \\
\hline 3829 & ${ }^{5} \mathrm{D}_{1}$ & vw \\
\hline 4007 & ${ }^{5} \mathrm{D}_{1}$ & vw \\
\hline 4027 & ${ }^{5} \mathrm{D}_{1}$ & vw \\
\hline $4353^{d}$ & ${ }^{5} D_{l}$ & $w$ \\
\hline $4570^{d}$ & ${ }^{5} D_{l}$ & $w$ \\
\hline $4797^{d}$ & ${ }^{5} D_{l}$ & $w$ \\
\hline 4817 & ${ }^{5} \mathrm{D}_{1}$, abs & $\mathrm{m}, \mathrm{w}$ \\
\hline 4910 & abs & s \\
\hline 4924 & abs & s \\
\hline $5055^{c, e}$ & $a b s$ & $v w$ \\
\hline $5074^{c, e}$ & $a b s$ & $v w$ \\
\hline 5091 & abs & $\mathrm{w}$ \\
\hline 5111 & abs & $\mathrm{vw}$ \\
\hline 5135 & abs & vw \\
\hline 5209 & abs & vw \\
\hline 17283 & ${ }^{5} D_{0}$, abs, exc & vw \\
\hline 19025 & abs, exc & $\mathbf{m}, \mathbf{m}$ \\
\hline 19038 & abs, exc & $\mathrm{w}, \mathrm{w}$ \\
\hline 19053 & abs, exc & $\mathbf{w}, \mathbf{w}$ \\
\hline $19065^{c, e}$ & exc & $w$ \\
\hline 21449 & exc & $\mathbf{m}$ \\
\hline 21467 & abs, exc & $\mathbf{v w}, \mathbf{m}$ \\
\hline 21516 & abs, exc & $\mathbf{w}, \mathbf{m}$ \\
\hline 21519 & abs & $\mathrm{vw}$ \\
\hline $21542^{d}$ & exc & $w$ \\
\hline 21565 & abs, exc & $\mathbf{w}, \mathbf{m}$ \\
\hline
\end{tabular}

${ }^{a}$ The initial selection of the lines is shown in bold. Lines not included in the fitting are printed in italic. A complete list of the identified transitions is available in the Supporting Information (Table S1). ${ }^{b}$ Key: vs, very strong; s, strong; m, medium; w, weak; vw, every weak; ${ }^{5} \mathrm{D}_{0}$, emission from ${ }^{5} \mathrm{D}_{0} ;{ }^{5} \mathrm{D}_{1}$, emission from ${ }^{5} \mathrm{D}_{1}$; ${ }^{5} \mathrm{D}_{2}$, emission from ${ }^{5} \mathrm{D}_{2}$. ${ }^{c}$ Excessive line. ${ }^{d}$ Line located in the interband gap. ${ }^{e}$ Phonon sideband.

6 is slightly different. From 5 to $70 \mathrm{~K}$ the lifetime of the ${ }^{5} \mathrm{D}_{2}$ rises from 24.4 to $27.7 \mu \mathrm{s}$, then it starts to be shorter exponentially to $8.3 \mu \mathrm{s}$ at $300 \mathrm{~K}$.

Quenching of Luminescence Energies. Figures 7 and 8 present the Arrhenius plots ${ }^{56}$ that represent the influence of

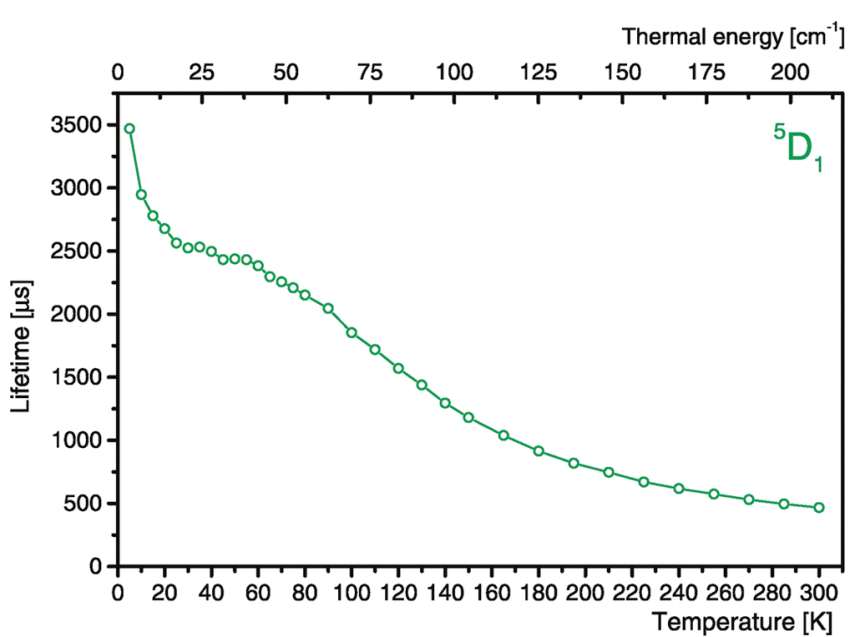

Figure 5. Influence of temperature on the lifetime of the ${ }^{5} \mathrm{D}_{1}$ multiplet of $\mathrm{Eu}^{3+}$ in $\mathrm{K}_{5} \mathrm{Li}_{2} \mathrm{EuF}_{10}$ crystal.

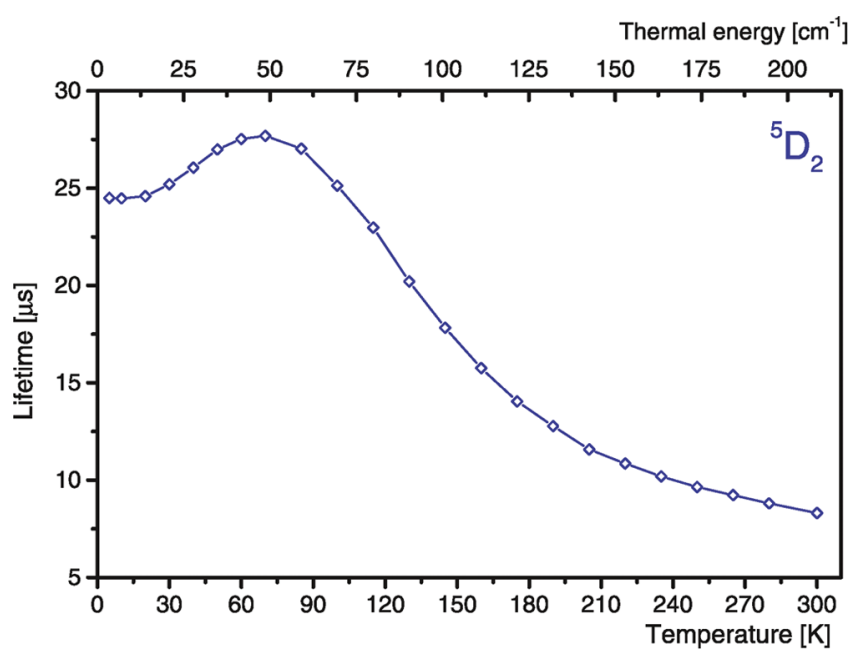

Figure 6. Influence of temperature on the lifetime of the ${ }^{5} \mathrm{D}_{2}$ multiplet of $\mathrm{Eu}^{3+}$ in $\mathrm{K}_{5} \mathrm{Li}_{2} \mathrm{EuF}_{10}$ crystal.

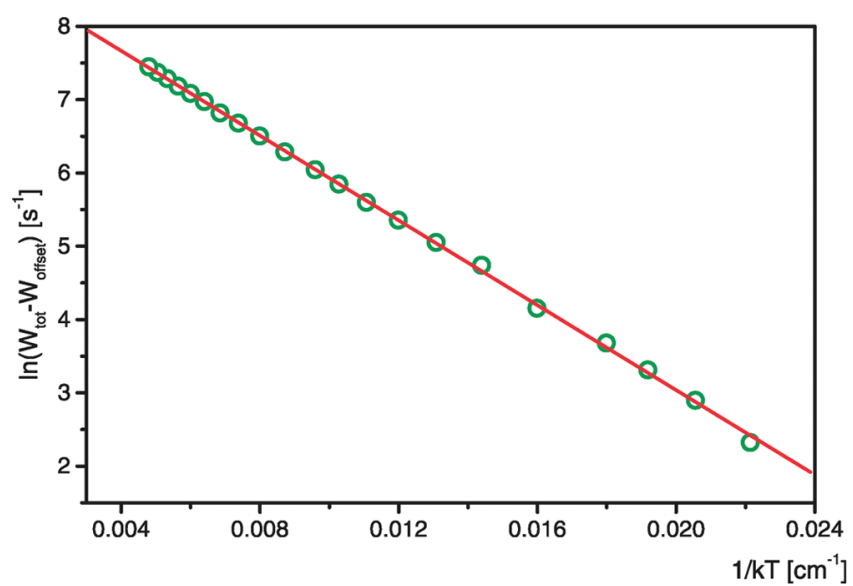

Figure 7. Arrhenius' fit to the temperature-dependent nonradiative decay rate of the ${ }^{5} \mathrm{D}_{1}$ multiplet in $\mathrm{K}_{5} \mathrm{Li}_{2} \mathrm{EuF}_{10}$. An activation energy of $-296 \mathrm{~cm}^{-1}$ of the process of emission quenching is obtained.

thermal energy onto the ${ }^{5} \mathrm{D}_{1}$ (Figure 7) and ${ }^{5} \mathrm{D}_{2}$ (Figure 8) transition speeds.

$$
\ln (W)=\ln (A)-\frac{E_{\mathrm{q}}}{k T}
$$




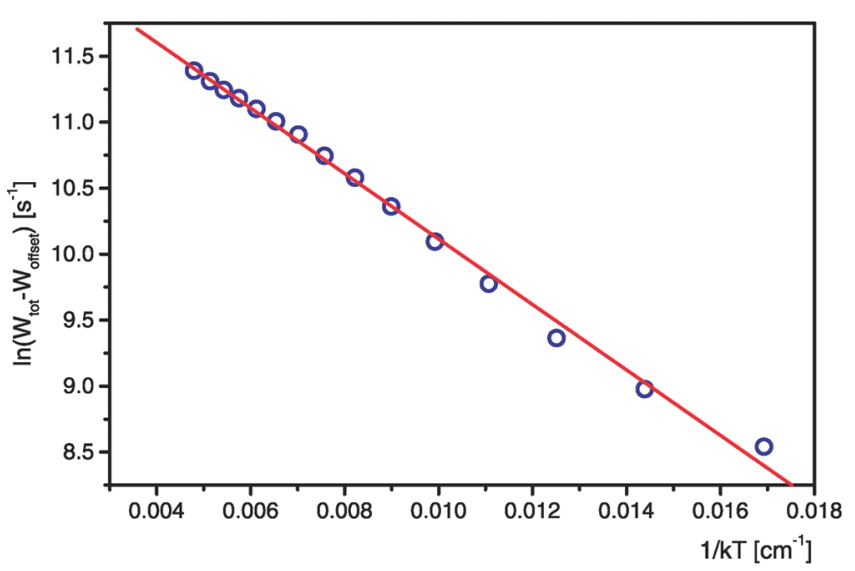

Figure 8. Arrhenius' fit to the temperature-dependent nonradiative decay rate of the ${ }^{5} \mathrm{D}_{2}$ multiplet in $\mathrm{K}_{5} \mathrm{Li}_{2} \mathrm{EuF}_{10}$. An activation energy of $-248 \mathrm{~cm}^{-1}$ of the process of emission quenching is obtained.

where $W$ is the transition rate and $A\left(s^{-1}\right)$ represents a transition speed given by the intercept of the line at $1 / T \rightarrow 0$ and is call the pre-exponential factor. The parameter $E_{\mathrm{q}}$ (quenching energy, $(\mathrm{cm}))$ is obtained from the slope of the line.

For the thermal quenching of the ${ }^{5} \mathrm{D}_{1}$ multiplet we obtained $\ln (A)=8.864$ and $E_{\mathrm{q}}=296.00 \mathrm{~cm}^{-1}$. The values representing the quenching of the ${ }^{5} \mathrm{D}_{2}$ state were found to be 12.595 and $248.07 \mathrm{~cm}^{-1}$, respectively. These data evidence that in both kinds of quenching the ${ }^{7} \mathrm{~F}_{1}$ multiplet is involved with its energies of about $300 \mathrm{~cm}^{-1}$; see Table 2 .

Fitting the Experimental Data: Energy Levels and Parameters. All the interactions in Hamiltonian (1) have been diagonalized simultaneously in the $\mathrm{SLM}_{J}$ function basis restricted to the lowest 1950 of 3003 states. This restriction has in practice no influence on the position of the energy levels below $60000 \mathrm{~cm}^{-1}$. The calculations have been performed using the f-shell empirical programs provided by Reid. ${ }^{57}$

Two main phases of the fitting procedure relate to two various models here employed: the initial one, based on the EAOM approximation and called model $\mathrm{A}$, and the refined one, model B. The models differ in varying FI parameters and in parameters describing the $\mathrm{CF}$ effect, but they share the same assignation of the observed electronic transitions.

Model A. The known regularity of the free-ion parameters (FIP) ${ }^{15-17,28}$ justifies restriction of their variation to the main intraion interactions, namely, the Coulomb repulsion represented by the Slater integrals, $F^{k}, k=2,4,6$, and the spin-orbit coupling with its constant $\xi_{4 \mathrm{f}}$. All the remaining corrections of higher order in the perturbation expansion have been fixed at their literature values. ${ }^{16}$

The $J$ splittings of the lowest ${ }^{7} \mathrm{~F}$ and ${ }^{5} \mathrm{D}$ terms given by the barycenter's of the corresponding well separated groups of the observed lines below $25000 \mathrm{~cm}^{-1}$ determine the spin-orbit coupling constant, but not the Slater parameters. Thus, transitions above $25000 \mathrm{~cm}^{-1}$ have to be included, preferably, the most intense lines or less intense but located in relatively well resolved bands or in vicinity of the interband gaps. In this way we selected 58 transitions the energies of which are distinguished in Table 2 with the bold typeface.

The starting FIP were taken from ref 16 and CF effective potential was approximated by the single-parameter model EAOM-I defined in section. The initial value of $e_{\sigma}$ was (435 $\mathrm{cm}^{-1}$ ) reported for $\mathrm{Tb}^{3+}$ in $\mathrm{LiTbF}_{4}{ }^{58}$ The five-parameter model (four FI parameters and one EAOM-I parameter) applied first allowed us to assign and describe all the 58 electronic lines fairly well with the root mean squared error (rmse) of $20.3 \mathrm{~cm}^{-1}$ and the largest error below $50 \mathrm{~cm}^{-1}$. The solution obtained with EAOM-I was employed then as an initial one in the next step, in which the general, three-parameters EAOM was applied. Within this step rmse was reduced to $15.1 \mathrm{~cm}^{-1}$, and the results were regarded to be reliable enough to serve as an assignation template for all the observed transitions listed in Table 2.

From the point of view of model calculations either the excluded weak lines are the excessive transitions or they lie in the characteristic for $\mathrm{Eu}^{3+}$ spectral gaps. ${ }^{16,25,59} \mathrm{We}$ found that forced inclusion of the latter group results in the maximum error exceeding considerably the $50 \mathrm{~cm}^{-1}$ limit even if the general all-parameters approach (see model B) was applied. The error for these lines could not be reduced below this limit even for reduced number of the transitions included in the fitting to these of "s" and "vs" intensities (see Table 2) which lay below, subsequently, 27000,25000 , and $22000 \mathrm{~cm}^{-1}$. Origin of these lines remains unclear although their low intensity and characteristic shifts from the main bands suggest presence of the mentioned hydrated surface phases.

The EAOM fit for the 160 transitions gives the rms error of $15.4 \mathrm{~cm}^{-1}$, differing only by $0.3 \mathrm{~cm}^{-1}$ from that for 58 transitions, what points to a similar quality of the assignation of the additional 102 lines. The results are shown as the model A in Table 3 where apart from the obtained $e_{\mu}$ parameters also the corresponding $B_{k q}$ parameters are given.

They were calculated from the $e_{\mu}$ parameters and the $W_{k q}^{u}$ coefficients listed in Table 2 according to the formula combining eqs 6,7 , and 10),

$$
B_{k q}=\left(B_{k q}\right)_{\mathrm{OA}}+\sum_{\mu} W_{k q}^{\mu} e_{\mu}
$$

and the rotation of the coordinate system determined by (4) and the standardization requirement (i).

At that point we can compare the EAOM calculations with the results obtained using DST (not presented). The fitting of the distinguished 58 lines with the five CF parameters of the Hamiltonian of approximated $D_{2 d}$ symmetry has led to the rms error of $16.7 \mathrm{~cm}^{-1}$, i.e., about $10 \%$ larger than that of EAOM with only three AOM parameters. Moreover, the errors attributed to individual energy levels are especially large just for degenerate ones in the DST model. One of them exceeds $40 \mathrm{~cm}^{-1}$ with the corresponding splitting of the degenerate level of about 64 $\mathrm{cm}^{-1}$. Note that, due to the nonlocal polarization effects and the influence of the whole crystal from outside of the coordination polyhedron, the low symmetry perturbation may be larger (or smaller) than that generated by the local interaction of the closest neighborhood. Those unpredictable in general nonlocal contributions have been taken into account explicitly in EAOM.

Model B. Having the standardized set of the $B_{k q}$ parameters obtained in model A and the preliminary assignation of all the identified transitions we are at point to apply the exact oneelectron parametrization of the CF interaction given by the expansion (3). The Rajnak and Wybourne correction to the Coulomb repulsion due to the interconfiguration interaction, ${ }^{14}$ with the parameters $\alpha, \beta$, and $\gamma$ has been adjusted at this phase as well. The higher-order corrections given from the third line of the Hamiltonian (2) have been kept fixed on their initial values similarly as in model A. Their adjustment in the case of such a large number of experimental energy levels would be possible but not necessarily tenable if applied without the correlation crystal field effect (see ref 31 and the references therein). The latter correction is expected to be of the same order. In its general form it multiplies the number of parameters. 
TABLE 3: Free-Ion and Standardized Crystal Field Parameters for $\mathrm{Eu}^{3+}$ Obtained in Models A and B (See Main Text $)^{a}$

\begin{tabular}{|c|c|c|}
\hline & model A & model B \\
\hline$E_{\mathrm{avg}}$ & $63752(5)$ & $63770(3)$ \\
\hline$F^{2}$ & $82695(17)$ & $82769(12)$ \\
\hline$F^{4}$ & $59439(38)$ & $59574(27)$ \\
\hline$F^{6}$ & $42232(23)$ & $42297(24)$ \\
\hline$\alpha$ & 20.2 & $20.2(0.1)$ \\
\hline$\beta$ & -567 & $-561(7)$ \\
\hline$\gamma$ & 1500 & $1454(6)$ \\
\hline$\xi$ & $1327(1)$ & $1327(1)$ \\
\hline$T^{2}$ & 300 & 300 \\
\hline$T^{3}$ & 40 & 40 \\
\hline$T^{4}$ & 60 & 60 \\
\hline$T^{6}$ & -300 & -300 \\
\hline$T^{7}$ & 370 & 370 \\
\hline$T^{8}$ & 320 & 320 \\
\hline$B_{20}$ & 300 & $305(16)$ \\
\hline$B_{22}$ & 32 & 139(10) \\
\hline$B_{40}$ & -13 & $260(30)$ \\
\hline$B_{42}$ & 1 & $-168(61)$ \\
\hline$B_{44}$ & 1240 & 1198(40) \\
\hline$B_{41}$ & -30 & $-15(66)$ \\
\hline$B_{43}$ & 23 & $365(130)$ \\
\hline$B_{60}$ & -589 & $-548(81)$ \\
\hline$B_{62}$ & 3 & $-29(34)$ \\
\hline$B_{64}$ & 306 & $276(45)$ \\
\hline$B_{66}$ & 12 & $133(27)$ \\
\hline$B_{61}$ & -160 & $-228(123)$ \\
\hline$B_{63}$ & -43 & $-144(61)$ \\
\hline$B_{65}$ & -40 & $99(46)$ \\
\hline$M^{0}$ & 2 & 2 \\
\hline$M^{2}$ & 1.12 & 1.12 \\
\hline$M^{4}$ & 0.62 & 0.62 \\
\hline$P^{2}$ & 360 & 360 \\
\hline$P^{4}$ & 180 & 180 \\
\hline$P^{6}$ & 36 & 36 \\
\hline$e_{\sigma}$ & $546(18)$ & \\
\hline$e_{\pi}$ & $272(16)$ & \\
\hline$e_{\delta}$ & $112(11)$ & \\
\hline no. of data points & 160 & 160 \\
\hline no. of free param & 8 & 22 \\
\hline rms error & 15.4 & 9.2 \\
\hline
\end{tabular}

${ }^{a}$ Errors of the varied parameters are given in parentheses. The $B_{k q}$ parameters in model A (printed in italic) have been calculated from the AOM parameters $e_{\mu}$ listed here and the data from Table 1 using eq 16.

Taking into account a margin of uncertainty connected with unassigned lines (listed in Table 2), such a tuning of the fitting would not be reasonable at the present stage.

Thus, at the final phase, the seven FI parameters and 14 ordinary $B_{k q}$ parameters were varied. The rms error was reduced to $9.2 \mathrm{~cm}^{-1}$. The results are shown in Tables $3-5$.

The $B_{k q}$ parameters displayed in Table 3 are in the standardized form, consistent with those obtained in model A according to the standardization requirement (ii). As seen, the leading parameters of each $k$-rank, namely, $B_{20}, B_{44}$, and $B_{60}$ are welldetermined and similar in these two models. Only these parameters, the absolute values of which are relatively small, e.g., lower than $100 \mathrm{~cm}^{-1}$ and additionally $B_{61}$, remain purely determined. $B_{44}$ is dominating among all the parameters in both models. It is interesting to notice a similar observation for the uranium ion in $\mathrm{K}_{5} \mathrm{Li}_{2} \mathrm{UF}_{10} \cdot{ }^{60}$ Moreover, the crystal field strength parameter $N_{v}$ defined as ${ }^{39,40}$

$$
N_{v}=\left[4 \pi \sum_{k q} \frac{1}{2 k+1}\left(B_{k q}\right)^{2}\right]^{1 / 2}
$$

is for the uranium $\left(4334 \mathrm{~cm}^{-1}\right)$ twice as large as for europium $\left(2349 \mathrm{~cm}^{-1}\right)$, in nice agreement with the systematics presented for a wide range of actinide and lanthanide compounds. ${ }^{61}$ The

TABLE 4: Experimental and Computed (Model B) Energy Levels for $\mathrm{Eu}^{3+}$ in $\mathrm{K}_{5} \mathrm{Li}_{2} \mathrm{LaF}_{10}$ below $21600 \mathrm{~cm}^{-1}$ a

\begin{tabular}{|c|c|c|c|}
\hline SLJ state ${ }^{b}$ & $\mathrm{fit}^{c}$ & observed & difference \\
\hline${ }^{7} \mathrm{~F}_{0}$ & 3 & 0 & -3 \\
\hline \multirow[t]{3}{*}{${ }^{7} F_{1}$} & 309 & 313 & 4 \\
\hline & 387 & 377 & -10 \\
\hline & 438 & 429 & -9 \\
\hline \multirow[t]{5}{*}{${ }^{7} \mathrm{~F}_{2}$} & 868 & 900 & 32 \\
\hline & 1005 & 1010 & 5 \\
\hline & 1062 & 1055 & -7 \\
\hline & 1092 & 1089 & -3 \\
\hline & 1149 & 1155 & 6 \\
\hline \multirow{7}{*}{${ }^{7} \mathrm{~F}_{3}$} & 1830 & 1832 & 2 \\
\hline & 1863 & 1858 & -5 \\
\hline & 1887 & 1884 & -3 \\
\hline & 1902 & 1905 & 3 \\
\hline & 1973 & 1970 & -3 \\
\hline & 1993 & 2006 & 13 \\
\hline & 2012 & 2035 & 24 \\
\hline \multirow[t]{9}{*}{${ }^{7} \mathrm{~F}_{4}$} & 2711 & 2719 & 8 \\
\hline & 2760 & 2776 & 16 \\
\hline & 2788 & 2795 & 7 \\
\hline & 2794 & 2806 & 12 \\
\hline & 2927 & 2919 & -8 \\
\hline & 2951 & 2954 & 3 \\
\hline & 3017 & 3014 & -3 \\
\hline & 3042 & 3034 & -8 \\
\hline & 3096 & & \\
\hline \multirow[t]{11}{*}{${ }^{7} \mathrm{~F}_{5}$} & 3812 & & \\
\hline & 3814 & 3815 & 1 \\
\hline & 3816 & & \\
\hline & 3827 & 3829 & 2 \\
\hline & 3926 & & \\
\hline & 3945 & & \\
\hline & 4015 & 4007 & -8 \\
\hline & 4031 & 4027 & -4 \\
\hline & 4037 & & \\
\hline & 4075 & & \\
\hline & 4123 & & \\
\hline \multirow[t]{13}{*}{${ }^{7} \mathrm{~F}_{6}$} & 4832 & 4817 & -15 \\
\hline & 4834 & & \\
\hline & 4918 & 4910 & -8 \\
\hline & 4920 & 4914 & -6 \\
\hline & 5099 & 5091 & -8 \\
\hline & 5117 & 5111 & -6 \\
\hline & 5138 & 5135 & -3 \\
\hline & 5172 & & \\
\hline & 5192 & & \\
\hline & 5202 & & \\
\hline & 5204 & 5209 & 5 \\
\hline & 5222 & & \\
\hline & 5228 & & \\
\hline${ }^{5} \mathrm{D}_{0}$ & 17292 & 17283 & -9 \\
\hline \multirow[t]{3}{*}{${ }^{5} \mathrm{D}_{1}$} & 19015 & 19025 & 10 \\
\hline & 19036 & 19038 & 2 \\
\hline & 19056 & 19053 & -3 \\
\hline \multirow{5}{*}{${ }^{5} \mathrm{D}_{2}$} & 21461 & 21449 & -12 \\
\hline & 21500 & 21467 & -33 \\
\hline & 21508 & 21516 & 8 \\
\hline & 21515 & 21519 & 4 \\
\hline & 21542 & 21565 & 23 \\
\hline
\end{tabular}

${ }^{a}$ The extended results of the fitting up to $60000 \mathrm{~cm}^{-1}$ have been collected in the Supporting Information (Table S2). ${ }^{b}$ The leading one or two eigenvector components are shown. ${ }^{c}$ The parameters used to compute these levels are given in Table 3, model B. ${ }^{d}$ Gaps in the energy levels structure above $28000 \mathrm{~cm}^{-1}$ are collected in Table 5. 
TABLE 5: Gaps Larger Than $300 \mathrm{~cm}^{-1}$ in the Model Energy Levels Structure above $28000 \mathrm{~cm}^{-1}$

\begin{tabular}{lc}
\hline \multicolumn{1}{c}{ energy range } & energy gap \\
\hline $28810\left({ }^{5} \mathrm{~L}_{10}\right)-30798\left({ }^{5} \mathrm{H}_{3}\right)$ & 1988 \\
$31755\left({ }^{5} \mathrm{H}_{6}\right)-32836\left({ }^{3} \mathrm{P}_{0}\right)$ & 1081 \\
$33633\left({ }^{5} \mathrm{~F}_{4}\right)-33998\left(\left({ }^{5} \mathrm{I}_{4}\right)\right.$ & 365 \\
$35654\left({ }^{5} \mathrm{I}_{7}\right)-36150\left({ }^{5} \mathrm{~K}_{5}\right)$ & 496 \\
$36395\left({ }^{5} \mathrm{~K}_{5}\right)-37303\left({ }^{5} \mathrm{~K}_{6}\right)$ & 909 \\
$37514\left({ }^{5} \mathrm{~K}_{6}\right)-38154\left({ }^{3} \mathrm{P}_{1}\right)$ & 640 \\
$40484\left({ }^{5} \mathrm{D}_{3}+{ }^{5} \mathrm{G}_{5}\right)-40888\left({ }^{3} \mathrm{O}_{10}\right)$ & 404 \\
$43702\left({ }^{3} \mathrm{M}_{9}\right)-44001\left({ }^{3} \mathrm{O}_{12}\right)$ & 299 \\
$45101\left({ }^{3} \mathrm{M}_{10}\right)-45433\left({ }^{3} \mathrm{~F}_{4}\right)$ & 333 \\
$45489\left({ }^{3} \mathrm{~F}_{4}\right)-45788\left({ }^{5} \mathrm{P}_{2}+{ }^{5} \mathrm{P}_{1}\right)$ & 298 \\
$47136\left({ }^{3} \mathrm{~N}_{9}\right)-47559\left({ }^{3} \mathrm{H}_{5}\right)$ & 423 \\
$48317\left({ }^{3} \mathrm{G}_{5}\right)-48961\left({ }^{3} \mathrm{~K}_{8}\right)$ & 644 \\
$49260\left({ }^{3} \mathrm{D}_{2}+{ }^{3} \mathrm{~K}_{8}\right)-49570\left({ }^{3} \mathrm{D}_{1}\right)$ & 310 \\
$51033\left({ }^{3} \mathrm{~K}_{7}\right)-51649\left({ }^{3} \mathrm{H}_{4}+{ }^{3} \mathrm{H}_{6}\right)$ & 616 \\
$53340\left({ }^{3} \mathrm{~K}_{8}\right)-53817\left({ }^{3} \mathrm{I}_{6}\right)$ & 478 \\
$54160\left({ }^{3} \mathrm{M}_{10}\right)-54515\left({ }^{3} \mathrm{I}_{7}\right)$ & 355 \\
$54640\left({ }^{5} \mathrm{~S}_{2}\right)-54942\left({ }^{3} \mathrm{H}_{6}\right)$ & 302
\end{tabular}

parameters of sixth rank, contrary to the fourth rank ones, are especially small in absolute values. The geometrical factors shown in Table 1 point to specific arrangement of the eight ligating fluorine ions, leading to the mutual cancellation of the $\sigma$ and $\pi$ contributions for these parameters. To verify our solution, we probed several sets of initial parameters generated randomly for the actual and various, more restricted choices of experimental lines mentioned above. The calculations led to different stable solutions but none of them gave lower rms error than that obtained within the model B. They probably correspond to local minima in the multidimensional parametric space.

The largest error, exceeding $30 \mathrm{~cm}^{-1}$, is seen in Table 4 for the two lines: $900 \mathrm{~cm}^{-1}\left({ }^{7} \mathrm{~F}_{2}\right)$ deduced from the emission patterns and $21467 \mathrm{~cm}^{-1}\left({ }^{5} \mathrm{D}_{2}\right)$ observed in excitation spectra. The latter resembles the structure at $21542 \mathrm{~cm}^{-1}$, that was not included in the fitting at the EAOM phase. On the basis of the absorption data solely, another assignment with the double structure of the line $21517 \mathrm{~cm}^{-1}$ and only one line around $21450 \mathrm{~cm}^{-1}$ could be acceptable and consistent in both models. Nevertheless, the intensity and quality of the excitation spectra in the same region ground what has been accepted.

The majority of the observed transitions have been simulated with accuracy below $6 \mathrm{~cm}^{-1}$. Such a value can be considered as a limit of exactness, taking into account precision of the location of the experimental lines and accuracy of the employed model itself. Quality of the fitting of the lowest 101 of all 160 observed lines is even better: more lines are reproduced with accuracy below and above limit despite complete or almost complete sequences of experimental levels in this region. On the other hand, most of transitions of higher energies, above $28000 \mathrm{~cm}^{-1}$, are excluded by the selection rules for the radiation field since the states become rich of components with high values of the angular momentum and the magnetic quantum numbers. Many of these which can be observed lie at the vicinity of or at the edges of the interband gaps so their flexibility in assignation is limited. The FI parameters in the two models were stable during the fitting, varying in a narrow range.

They are also consistent with the initial values taken from the classical paper by Carnall et al. ${ }^{16}$

The positions of the interband gaps shown in Table 5 relate to those reported for $\mathrm{Eu}^{3+}$ in $\mathrm{LaF}_{3},{ }^{16}$ but their magnitudes are lower by a value of about $100 \mathrm{~cm}^{-1}$. This suggests a stronger crystal field in relation to $\mathrm{Eu}^{3+}$ in $\mathrm{LaF}_{3}$. Indeed, the parameters themselves or more precisely, the CF strength factors calculated from (17) for the two compounds, namely 2349 and $2152 \mathrm{~cm}^{-1}$, respectively, confirm the expectation.

\section{Conclusions}

This work reports spectra and their model description of belonging to family of uncommon stoichiometric luminescence compound $\mathrm{K}_{5} \mathrm{Li}_{2} \mathrm{LnF}_{10}{ }^{6}$ where Ln denotes: $\mathrm{Ce}, \mathrm{Nd}, \mathrm{Pr}$, Eu, and $\mathrm{Gd}$. It was found that in these crystals, concentration quenching of luminescence is unusually weak, with some exceptions: ${ }^{1} \mathrm{D}_{2}$ level of $\mathrm{Pr}^{3+}$ or ${ }^{5} \mathrm{D}_{3}$ level of $\mathrm{Eu}^{3+}$.

The emission lines from the ${ }^{5} \mathrm{D}_{1}$ and ${ }^{5} \mathrm{D}_{0}$ states in KLEF is overlapped and causes difficulties in determining the ${ }^{7} \mathrm{~F}_{J}$ Stark sublevels. At $5 \mathrm{~K}$ the lifetimes of emission originated in the ${ }^{5} \mathrm{D}_{0}$ and ${ }^{5} \mathrm{D}_{1}$ are 9.5 and $3.5 \mathrm{~ms}$, respectively. In KLEF luminescence of the ${ }^{5} \mathrm{D}_{1}$ and ${ }^{5} \mathrm{D}_{2}$ multiplets is thermally dependent as opposed to the ${ }^{5} \mathrm{D}_{0}$ one that was helpful in assignment of the emission line based on spectra recorded at higher temperatures. The quenching energies of the ${ }^{5} \mathrm{D}_{1}$ and ${ }^{5} \mathrm{D}_{2}$ multiplets were determined to be about $300 \mathrm{~cm}^{-1}$ and might suggest that in this processes the ${ }^{7} F_{1}$ level is involved. Although the local symmetry of the $\mathrm{Eu}^{3+}$ ion in this material is rather low, the intensity of the hypersensitive transitions in the whole family of $\mathrm{K}_{5} \mathrm{Li}_{2} \mathrm{LnF}_{10}$ crystals, especially for $\mathrm{Eu}^{3+}$ the ${ }^{7} \mathrm{~F}_{0} \rightarrow$ ${ }^{5} \mathrm{D}_{2}$ one, is very low.

Despite apparent simplicity of the lowest, well separated ${ }^{7} \mathrm{~F}_{J}$ and ${ }^{5} \mathrm{D}_{J}$ bands and the ${ }^{7} \mathrm{~F}_{0}$ ground state that does not split in the crystal field, $\mathrm{Eu}^{3+}$ is not an easy ion for a model analysis of the optical properties. Its exceptional sensitivity to the crystal surrounding and the strong $J$-mixing, characteristic for dense bands of lines of higher energies makes the conventional parametrization practically useless. The present study shows that even in the case of so low symmetry as in KLEF the CF details of the electronic structure of the 3003-fold degenerate $\mathrm{f}^{6}$ system seen in the optical spectra recorded up to the $\mathrm{f}-\mathrm{d}$ absorption edge at $58000 \mathrm{~cm}^{-1}$ are determinable in the frames of the multistep phenomenological approach in good agreement with the microscopic picture of the main interactions, which, in the case of insulating solids, are known fairly well. Ab initio calculations of certain crystal-specific contributions to the $\mathrm{CF}$ effective potential and the simple AOM parametrization of the complex interaction of the nearest neighbor representing the remainder, in short, the enhanced angular overlap model, reduces drastically the space of possible solutions and allows one to control the physical sens of the parameters. EAOM has been employed to assign the observed transitions and to set up the final, refining fitting based on the general parametrization for the whole investigated energy range. Small rms error, stability of the solutions, and convergence of the standardized leading $B_{k q}$ parameters for the simplified model, A, and the refined one, $\mathrm{B}$, point to the reliability of the obtained results and open a way to similar investigations on other lanthanide ions, not only in this interesting matrix.

Acknowledgment. We thank Dr. Michelle Faucher (Clamart, France) for the CHLOE program, Dr. Michael F. Reid (University of Canterbury, Christchurch, New Zealand) for the f-shell programs, and Prof. Witold Ryba-Romanowski (ILT\&SR PAS Wrocław, Poland) for his kind interest in this work and inspiring discussions. The research leading to these results has received funding from the European Community's Seventh Framework Programme (FP7/2007-2013) under grant agreement $n^{\circ} 226716$, contract II-20090073 EC.

Supporting Information Available: List of all the observed transitions in absorption, emission, and excitation measurements 
(Table S1) and extended results (energy levels) of the fitting (Table S2). This material is available free of charge via the Internet at http://pubs.acs.org.

\section{References and Notes}

(1) Lempicki, A.; McCollum, B. C. J. Lumin. 1979, 20, 291.

(2) Dominiak-Dzik, G.; Gołab, S.; Bałuka, M.; Pietraszko, A.; Hermanowicz, K. J. Phys.: Condens. Matter 1999, 11, 5245.

(3) Sokólska, I.; Gołab, S.; Bałuka, M.; Ryba-Romanowski, W. J. Lumin. 2000, 91, 79. 1289.

(4) Solarz, P.; Ryba-Romanowski, W. J. Phys. Chem. Solids 2003, 64,

(5) Ryba-Romanowski, W.; Solarz, P. Chem. Phys. Lett. 2003, 27, 377.

(6) Solarz, P.; Ryba-Romanowski, W. Phys. Rev. B 2005, 72, 75105.

(7) Danielmeyer, P.; Weber, H. P. J. IEEE Quantum Electron 1972, 8,805 .

(8) Danielmeyer, H. G. Stoichiometric laser materials. In Festkorperprobleme (Advances in Solid State Physics); Quesser, H. J., Ed.; Pergamon: Vieweg, Braunschweig, Germany, 1975; Vol. XV.

(9) Judd, B. R. Phys. Rev. 1962, 127, 750.

(10) Ofelt, G. S. J. Chem. Phys. 1962, 37, 511

(11) Gajek, Z. Phys. Rev. B 2005, 72, 45139

(12) Hong, H.-P.; McCollum, B. Mater. Res. Bull. 1979, 14, 137.

(13) Gagor, A. Acta Crystallogr. 2009, E65, i82.

(14) Wybourne, B. G. Spectroscopic Properties of Rare Earths; Interscience: New York, 1965.

(15) Morrison, C.; Leavitt, R. Spectroscopic properties of triply ionized lanthanides in transparent host crystals. In Handbook of the Physics and Chemistry of Rare Earths; Gschneidner, K. A., Jr., Eyring, L., Eds.; NorthHolland: Amsterdam, 1982; Vol. 5.

(16) Carnall, W.; Goodman, G.; Rajnak, K.; Rana, R. J. Chem. Phys. 1989, 90,3443 .

(17) Gorller-Walrand, C.; Binnemans, K. Rationalization of CrystalField Parametrization. In Handbook on the Physics and Chemistry of Rare Earths; Gschneidner, K. A., Jr., Eyring, L., Eds.; North-Holland: Amsterdam, 1996; Vol. 23.

(18) Liu, G.; Jacquier, B. Rationalization of Crystal-Field Parametrization. In Springer Series in Materials Science; Springer with Tsinghua University Press: Beijing, 2005; Vol. 83.

(19) Newman, D.; Ng, B. Rep. Prog. Phys. 1989, 52, 699.

(20) Gorller-Walrand, C.; Huygen, E.; Binnemans, K.; Fluyt, L. J. Phys.: Condens. Matter 1994, 6, 7797.

(21) Karbowiak, M.; Zych, E.; Holsa, J. J. Phys.: Condens. Matter 2003, 15,2169 .

(22) Chen, X.; Liu, G. J. Solid State Chem. 2005, 178, 419.

(23) Gajek, Z.; Mulak, J.; Krupa, J. J. Solid State Chem. 1993, 107, 413

(24) Mech, A.; Gajek, Z.; Karbowiak, M.; Rudowicz, C. J. Phys.: Condens. Matter 2008, 20, 385205.
(25) Holsa, J.; Lastusaari, M.; Marysko, M.; Tukia, M. J. Solid State Chem. 2005, 178, 435.

(26) Jorgensen, C. K.; Pappalardo, R.; Schmidtke, H. H. J. Chem. Phys. 1963, 39, 401

(27) Gerloch, M.; Harding, J. H.; Wooley, G. Struct. Bonding 1981, 46,1 .

(28) Gajek, Z. J. Phys.: Condens. Matter 2000, 12, 415.

(29) Gajek, Z.; Mulak, J. J. Phys.: Condens. Matter 1992, 4, 427.

(30) Faucher, M.; Garcia, D. Phys. Rev. 1982, B26, 5451.

(31) Mulak, J.; Gajek, Z. The Effective Crystal Field Potential; Elsevier: Amsterdam, 2000.

(32) Zimmerer, G. Radiat. Meas. 2007, 42, 859, and references therein.

(33) Newman, D. J.; Ng, B. K. C., Eds.; Crystal Field Handbook; Cambridge University Press: Cambridge, U.K., 2000.

(34) Rudowicz, C. J. Chem. Phys. 1986, 84, 5045

(35) Rudowicz, C.; Chua, M.; Reid, M. Physica B 2000, 291, 327.

(36) Burdick, G. W.; Reid, M. F. Mol. Phys. 2004, 33, 59.

(37) Rudowicz, C. Mol. Phys. 1991, 74, 1159.

(38) Mulak, J.; Mulak, M. J. Phys A: Math. Gen. 2005, 3, 6081.

(39) Leavitt, R. J. Chem. Phys. 1982, 77, 1661.

(40) Auzel, F.; Malta, O. J. Phys. (Paris) 1983, 44, 201.

(41) Rudowicz, C. J. Phys. C 1985, 18, 1415.

(42) Edvardsson, S.; Klintenberg, M. J. Alloys Compd. 1998, 275, 230.

(43) Schmidt, P. C.; Weiss, A.; Das, T. P. Phys. Rev. 1979, B19, 5525.

(44) Faucher, M. Private communication, 1983.

(45) Jorgensen, C.; Judd, B. Mol. Phys. 1964, 8, 281.

(46) Henry, D.; Fellows, R.; Chopin, G. Coord. Chem. Rev. 1967, 18, 199.

(47) Mason, S.; Peacock, R.; Steward, B. Chem. Phys. Lett. 1974, 29, 149.

(48) Mason, S.; Peacock, R.; Steward, B. Mol. Phys. 1975, 30, 1829.

(49) Porcher, P.; Caro, P. J. J. Chem. Phys. 1978, 68, 4176.

(50) Gorller-Walrand, C.; Binnemans, K. Spectral Intensities of f-f Tansitions. In Handbook on the Physics and Chemistry of Rare Earths; Gschneidner, K. A., Jr., Eyring, L., Eds.; North-Holland: Amsterdam, 1998; Vol. 25, p 101.

(51) Porcher, P.; Caro, P. J. J. Chem. Phys. 1976, 65, 89.

(52) Solarz, P. Opt. Mater. 2008, 31, 114.

(53) Wang, F.; Zhang, Y.; Fan, X.; Wang, M. J. Mater. Chem. 2006, 16,1031 , and references therein.

(54) Krupa, J.; Queffelec, M. J. Alloys Compd. 1997, 250, 287.

(55) Wegh, R.; Donker, H.; Oskam, K.; Meijerink, A. J. Lumin. 1999. $82,93$.

(56) Arrhenius, S. Z. Phys. Chem. 1889, 4, 226.

(57) Reid, M. f-shell programs (private communication), 1997.

(58) Urland, W. Chem. Phys. Lett. 1981, 83, 116.

(59) Gajek, Z. Simulation of the the energy level scheme for $\mathrm{Eu}^{3+}$ in $\mathrm{LaF}_{3}$ from the data reported by Carnall et al. ${ }^{16}$

(60) Karbowiak, M.; Gajek, Z.; Drozdzynski, J. Chem. Phys. 2005, 310, 239.

(61) Barbanel, Y. A.; Penkin, M. V. Radiochemistry 2002, 44, 3323336.

JP9093468 